\section{Adipose-Derived Mesenchymal Stem Cells Responses to Different Doses of Gamma Radiation}

\author{
Shahram Taeb ${ }^{1 \oplus}$, Mohammad Amin Mosleh-Shiraz ${ }^{1,2}$, Abbas \\ Ghaderi3,4, Seyed Mohammad Javad Mortazavi5, Mahboo- \\ beh Razmkhah ${ }^{3 *} \odot$
}

\begin{abstract}
Background: The effects of radiation on the cellular compartments of the tumor microenvironment (TME) might be essential in radiotherapy outcomes.

Objective: We aimed to assess the effects of the different doses of gamma irradiation on viability, ABCA1 and MMP-9 expression in adipose-derived mesenchymal stem cells (ASCs) as a critical part of TME.

Material and Methods: In this experimental study, ASCs were extracted from five healthy donors and irradiated with different doses of 5, 10 and 30 Gy of gamma. Then, RNA was extracted from irradiated ASCs and cDNA was synthesized. The viability of ASCs was determined at 24, 48, 72 and $168 \mathrm{~h}$ after irradiation using trypan blue staining. The expression of ABCA1 was checked by quantitative real-time (qRT)PCR technique and the expression of MMP-9 protein was evaluated by western-blot.

Results: Based on our findings, 10 Gy and 30 Gy but not 5 Gy of gamma irradiation significantly decreased the viability of ASCs after 24, 48, 72 and $168 \mathrm{~h}$ compared to the non-irradiated cells $(\mathrm{P}<0.05)$. However, a dose of 5 Gy increased ABCA1 in ASCs significantly compared to 10 Gy and $30 \mathrm{~Gy}$ ( $\mathrm{P}=0.01$ and $\mathrm{P}=0.02$, respectively). In addition, the analysis of western blot data showed that 5 Gy of gamma irradiation significantly increased the expression of MMP-9 in ASCs $(\mathrm{P}=0.019)$.
\end{abstract}

Conclusion: It is concluded that various doses of gamma radiation elicit differential ASCs responses that may lead to different tumor cell reactions to the radiotherapy through bystander effects.

Citation: Taeb Sh, Mosleh-Shiraz MA, Ghaderi A, Mortazavi SMJ, Razmkhah M. Adipose-Derived Mesenchymal Stem Cells Responses to Different Doses of Gamma Radiation. J Biomed Phys Eng. 2022;12(1):35-42. doi: 10.31661/jbpe.v0i0.1212.

Keywords

Radiation; Cancer; Stem Cells; Tumor Microenvironment; Bystander Effects

\section{Introduction}

Oumor microenvironment (TME) is considered as a dynamic and heterogeneous environment regulating cancer destiny and the outcome of cancer targeted therapies [1-3]. Among the cells that are present in TME, mesenchymal stem cells (MSCs) have impressively attracted great attention in recent years. MSCs are progenitor cells, which can be picked from numerous adult tissues such as adipose tissue, periodontal ligament, and lungs [4-6]. These cells produce various subsets of stromal renewing cells subscribed to maintain the diversity
${ }^{1} \mathrm{PhD}$, lonizing and Nonionizing Radiation Pro tection Research Center School of Paramedical Sciences, Shiraz University of Medical Sciences, Shiraz, Iran

${ }^{2} \mathrm{PhD}$, Department of Radio-oncology, Shiraz University of Medical Sciences, Shiraz, Iran

${ }^{3} \mathrm{PhD}$, Shiraz Institute for Cancer Research, School of Medicine, Shiraz University of Medical Sciences, Shiraz, Iran

${ }^{4} \mathrm{PhD}$, Department of Immunology, School of Medicine, Shiraz University of Medical Sciences, Shiraz, Iran

${ }^{5} \mathrm{PhD}$, Department of Medical Physics and Engineering, Shiraz Univer sity of Medical Science Shiraz, Iran

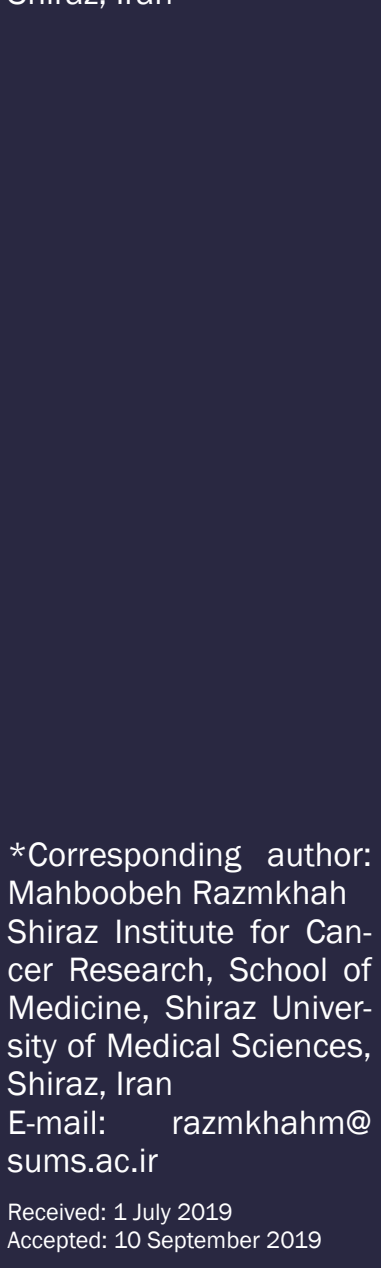


of tissues in the body [7, 8]. Moreover, MSCs are proficient in regulating the immune system by secreting several growth factors and cytokines [9]. Previous studies showed the presence of MSCs in the stromal of numerous tissues experiencing tumorigenesis and cancer development [10]. Nevertheless, other reports showed that MSCs have an anti-cancer role in the TME $[11,12]$.

For many years, scientists focused only on strategies targeting tumor cell but it seems to be not efficient enough. However, extending the therapeutic approaches to the TME may lead to the complete success of overcoming cancer [13]. Thereafter, scientists realized that the effects of radiation on TME might be decisive for the outcome of radiotherapy [14, 15]. In this regard, a previous study reported that irradiation induces reactive oxygen species, thereby stimulating TME modifications towards transformation and activating cancer cells and inflammatory responses [15]. Another study reported that microenvironment helps glioblastoma stem-like cells to resist against radiation [16]. It seems that releasing a variety of inflammatory cytokines from cancer stem cell which are located in the TME may lead to radioresistance and, consequently, failure in radiation therapy [17].

Since the tumor microenvironment might be effective in radiotherapy outcomes, in this study, we decided to evaluate the effects of different doses of gamma irradiation on the survival of ASCs and the expression of ABCA1 and MMP-9 in these cells.

\section{Material and Methods}

\section{Ethical approval and sampling}

In this experimental study, all of the persons who participated in this study approved informed and voluntary research consent. The original protocol of this study was sought and granted by the ethical committee of Shiraz University of Medical Sciences (IR.SUMS. REC.1396. S.290). All members were assured that their information would be preserved confidential. We randomly selected five healthy women who referred to the MRI Hospital for mammoplasty.

\section{ASCs isolation and culture}

We obtained adipose samples and extracted ASCs using a previously reported method [18]. Briefly, the tissues were transferred to the laboratory in normal saline tubes and washed twice with phosphate buffered saline (PBS). At first, for improving digestion of adipose tissues with $0.2 \%$ collagenase type I (GIBCO, USA), they were fragmented with surgical scalpels. Then, the crushed samples were incubated at $37{ }^{\circ} \mathrm{C}$ for $40 \mathrm{~min}$. The suspension was centrifuged at $400 \mathrm{G}$ for $5 \mathrm{~min}$ and cultured in DMEM medium supplemented with $1 \%$ penicillin/streptomycin and $10 \%$ fetal calf serum (GIBCO, USA). Finally, the flasks enclosing primary cells were kept in incubators with a moist condition, $5 \%$ of $\mathrm{CO}_{2}$ and at temperature of $37^{\circ} \mathrm{C}$. When the confluence of the primary cells reached more than $90 \%$, the cells were transported to larger flasks with a new culture medium. In addition, flow cytometry test was done to check the type of the cells $t$ extracted.

\section{Irradiation geometry}

After growth of ASCs and increase the confluence of ASCs to $90 \%$ per flask, they were irradiated to gamma with a dose of 5,10 and 30 Gy (dose rate $0.28 \mathrm{~Gy} / \mathrm{min}$ ). We used a Theratron cobalt-60 therapy unit (MDS Nordion, Canada) in the Radiotherapy and Radiation Oncology Department of Namazi Hospital. In this study, ASCs were irradiated in the field of view of $35 \times 35 \mathrm{~cm}^{2}$. In addition, the distance between the radiation source and the ASCs was $80 \mathrm{~cm}$. At the same time during the irradiation of ASCs, the cells of the control group were placed in the same conditions outside the radiation field. After irradiation, to inhibit repair of DNA damage, the flasks containing ASCs were moved onto icebox and im- 
ASCs Responses to Gamma Irradiation

mediately relocated to the incubator.

The viability of ASCs after gamma irradiation

In order to compare the growth rate of ASCs following exposure to gamma irradiation emitted from Co 60 source, the viability was measured during the culture period (in the third passage). ASCs were irradiated with either 0 , 5, 10 or $30 \mathrm{~Gy}$, and then harvested, counted and subsequently $5 \times 10^{3}$ cell $/ \mathrm{cm}^{2}$ of them were seeded in 96 well plates and incubated for $24 \mathrm{~h}$ in the standard condition $\left(37^{\circ} \mathrm{C} ; 5 \% \mathrm{CO}_{2}\right)$. The cells count was performed in $24,48,72$ and $168 \mathrm{~h}$ after irradiation by trypan blue staining. In addition, counts for each cell were repeated twice a day on experimental samples for each condition. The mean of the cell number was calculated within a week for each sample; culture medium exchange was performed every 72 h using DMEM.

\section{Quantitative Real-Time PCR}

To find the effects of gamma irradiation on ABCA1, qRT-PCR method (Bio-Rad, USA) was done. Briefly, ASCs were washed with PBS and RNA was extracted using $1 \mathrm{ml}$ of cold RNX-Plus (Invitrogen, Germany). In order to investigate the precision of the RNA, optical densities of extracted RNA were read at 260 and $280 \mathrm{~nm}$ using a NanoDrop spectrophotometer (Wilmington, DE, USA). Then, cDNA was made by the cDNA synthesis kit according to the manufacturer's instruction (Fermentas, Canada). All reactions were measured by 2x SYBR Green Master Mix (Applied Biosystems, USA) according to the hotstart Jumpstart Taq DNA Polymerase enzyme (Sigma, USA). The $\beta$-actin gene was considered as the housekeeping gene and all qPCR reactions were repeated twice. The primers were designed by AllelID software (Oligo Perfect Designer, Invitrogen, USA).

Western blotting of MMP9

In this study, the expression of MMP9 was determined using western blotting assay. In brief, ASCs were washed twice with PBS and lysed with protein extraction buffer (RIPA buffer: 1\% NP-40, 0.1\% SDS, 1\% DOC, $25 \mathrm{mM}$ Tris- $\mathrm{HCl} \mathrm{pH} 7.4$ and $150 \mathrm{mM} \mathrm{NaCl}$ ). ASCs suspension was then centrifuged for $20 \mathrm{~min}$, $12,000 \mathrm{~g}$ at $4{ }^{\circ} \mathrm{C}$. Then, Bradford assay was done to quantify the protein concentration and standardize equal protein. Next, $70 \mu \mathrm{g}$ protein per lane containing the supernatants and the same amount of loading buffer was run on $12 \%$ SDS-PAGE gel for $90 \mathrm{~min}$ at $100 \mathrm{~V}$ and then transferred onto a Polyvinylidene Fluoride (PVDF) membrane. The membrane was then blocked with 5\% nonfat skim milk PBS-Tween 80 (Sigma, USA) overnight at $4{ }^{\circ} \mathrm{C}$. Then, the blot was washed four times with washing buffer $(0.1 \%$ Tween 80 in PBS) and exposed to rabbit primary antibodies to MMP9 (Abcam, Cambridge, MA) with agitation at room temperature for $1 \mathrm{~h}$. The blot was then washed four times and Goat anti-rabbit of MMP9 (Abcam, Cambridge, MA) was used as a secondary antibody. Protein bands were visualized using ECL Plus reagents (GE Healthcare/Amersham) according to the manufacturer's protocol. Scanned images of the western blots were quantified by Image lab v4.01. Normalized band intensity was produced using $\beta$-actin band intensity in relevant columns.

\section{Statistical analysis}

SPSS software version 21 and GraphPad Prism version 7 were used for data analysis and graph presentation, respectively. T-test and independent t-test were used to analyze the parametric data, and Mann-Whitney and Wilcoxon were used for analyzing the nonparametric data. $\mathrm{P}<0.05$ was considered significant.

\section{Results}

Effect of irradiation on the viability of ASCS

To evaluate the effects of gamma irradiation 
on the viability of ASCs, we exposed the cells to the various doses of $0,5,10$, and $30 \mathrm{~Gy}$ of gamma and then cell count was performed at the time points of $24,48,72$ and $168 \mathrm{~h}$ with trypan blue. At first, we recorded the viability of ASCs in the non-irradiated group after $24 \mathrm{~h}(88.94 \pm 1.22 \%), 48 \mathrm{~h}(90.62 \pm 0.9 \%), 72 \mathrm{~h}$ $(92.43 \pm 0.88 \%)$ and $168 \mathrm{~h}(97.6 \pm 0.19 \%)$. Analysis of data showed that after exposing to 5 Gy of gamma irradiation, there was no significant difference in the viability of ASCs after $24 \mathrm{~h}$ $(61.58 \pm 10.08 \%, \mathrm{P}>0.9), 48 \mathrm{~h}(58.03 \pm 5.94 \%$, $\mathrm{P}>0.9), 72 \mathrm{~h}(74.4 \pm 21.9 \%, \mathrm{P}>0.9)$, and $168 \mathrm{~h}$ $(80.75 \pm 4.47 \%, \mathrm{P}>0.9)$ in comparison to nonirradiated ASCs. Then, regarding to non-irradiated ASCs, we found that 10 Gy gamma-irradiation decreased the viability of ASCs after $24 \mathrm{~h}(28.86 \pm 9.59 \%, \mathrm{P}=0.01), 48 \mathrm{~h}(27.73 \pm$ $0.7 \%, \mathrm{P}=0.04), 72 \mathrm{~h}(34.73 \pm 3.26 \%, \mathrm{P}=0.08)$ and $168 \mathrm{~h}(46.53 \pm 3.39, \mathrm{P}=0.03)$. Similarly, viability of 30 Gy gamma-irradiated ASCs decreased significantly at $24 \mathrm{~h}(18.72 \pm 2.1 \%$, $\mathrm{P}=0.001), 48 \mathrm{~h}(14.02 \pm 2.1 \%, \mathrm{P}<0.0001), 72$ $\mathrm{h}(23.27 \pm 5.46 \%, \mathrm{P}<0.0001)$ and $168 \mathrm{~h}(26.42$ $\pm 9.2 \%, \mathrm{P}<0.0001$ ) after irradiation (Figure 1).
QRT-PCR analysis

To understand the effect of gamma radiation on the $\mathrm{ABC}$ transporter of ASCs, expression of ABCA1 was investigated $72 \mathrm{~h}$ after irradiation. As depicted in Figure 2, in comparison to non-irradiated ASCs, 10 Gy and 30 Gy of gamma irradiation decreased the expression of ABCA1. These changes were not statistically different $(\mathrm{P}=0.52$ and $\mathrm{P}=0.61$, respectively). However, the expressions of ABCA1 in 5 Gy irradiated ASCs were, respectively, 3.2 and 2.9 fold higher than 10 Gy and 30 Gy irradiated cells $(\mathrm{P}=0.01$ and $\mathrm{P}=0.02)$.

\section{Western blot analysis}

Western blot analysis was performed to examine the expression of MMP-9 in irradiated (IR) and non-irradiated (NIR) ASCs. Due to the upregulation of ABCA1 transcripts in 5 Gy of gamma irradiation, we checked the expression of MMP-9 in a dose of 5 Gy regarding non-irradiated ASCs. As shown in Figure 3, western blot analysis showed that a dose of 5 Gy significantly increased the expression of MMP-9 protein to 1.93 fold regarding non-

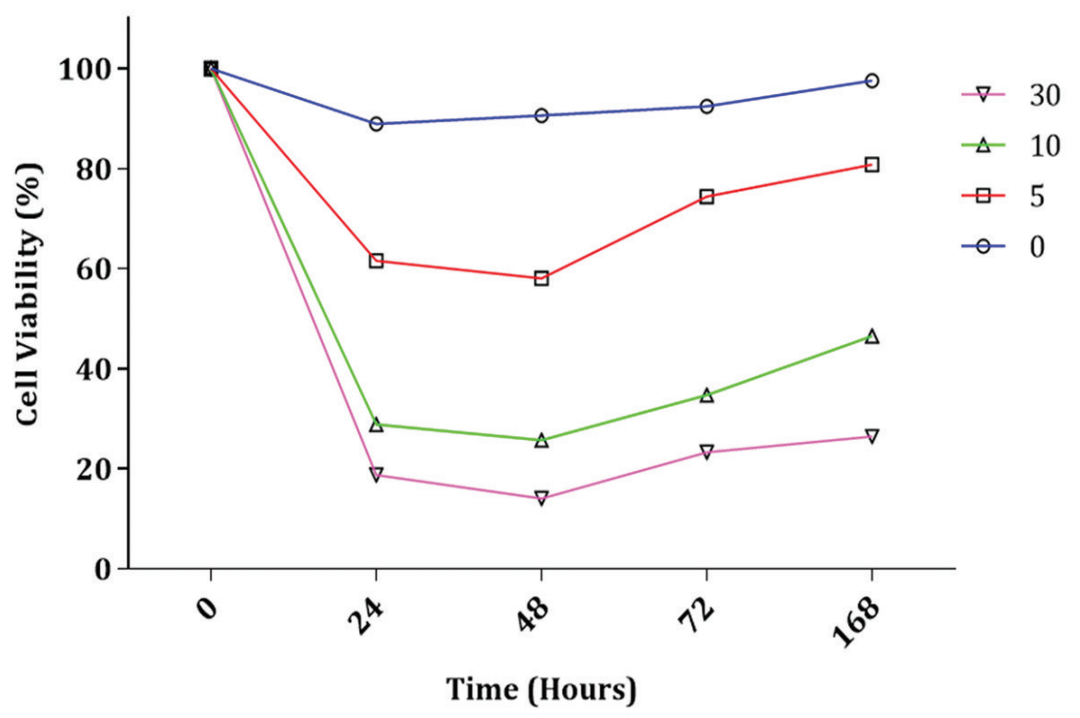

Figure 1: The percentage of cell viability in irradiated ASCs. After irradiating with 5, 10 and 30Gy of gamma irradiation, 5000 cells were cultured in a 96-well plate. Cell counting was done with trypan blue at $24,48,72$ and $168 \mathrm{~h}$ after exposure. The test was done as a duplicate and data are presented as mean. ASCs: Adipose-derived mesenchymal stem cells 


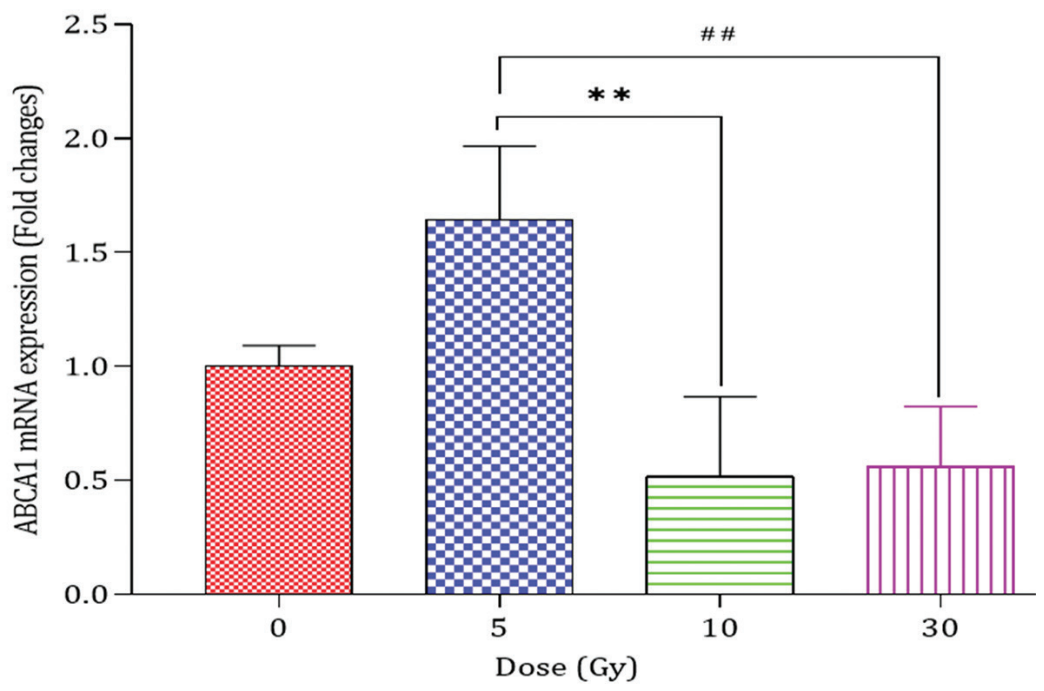

Figure 2: The mRNA expression of ABCA1 in ASCs after exposure to 5, 10 and 30 Gy of gamma irradiation. Each experiment was conducted twice independently, with each sample measured in duplicate. Error bars indicate the standard error of the mean and statistical significance was set at $\mathrm{P}<0.05\left({ }^{* *}\right)$. ASCs: Adipose-derived mesenchymal stem cells
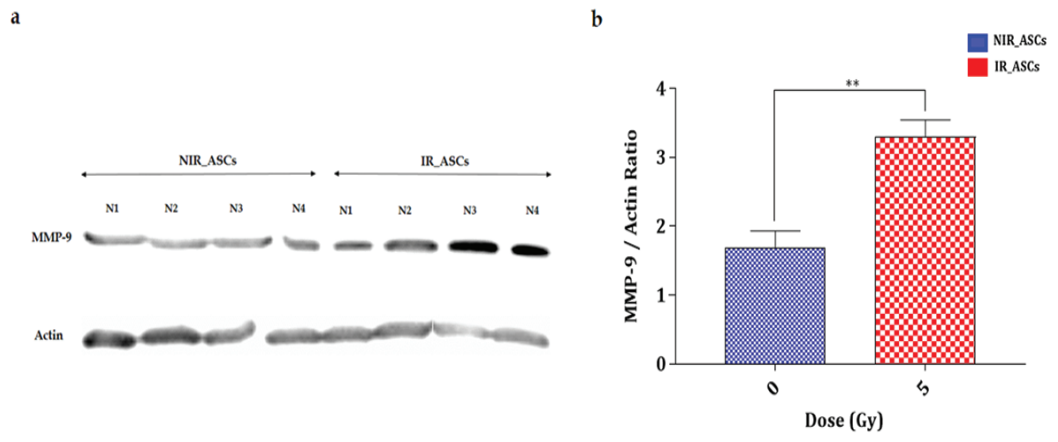

Figure 3: Detection of MMP-9 protein by western blot. a: The blot was subsequently probed with antibodies for MMP-9 and $\beta$-actin. b: Data analysis in the chart denoted the MMP-9 to the $\beta$-actin ratio in irradiated and non-irradiated ASCs. Data represents mean \pm SD and significant differences between groups are indicated as ** $(P<0.05)$. ASCs: Adipose-derived Mesenchymal Stem Cells. N1-4: Number of samples. IR-ASC: Irradiated ASCs, NIR: Non-Irradiated ASCs.

irradiated ASCs $(\mathrm{P}=0.019)$.

\section{Discussion}

The cellular compartment of the tumor site may affect the response rate of the tumor cells to the radiotherapy and different doses of radiation. In this study, we exhibited that exposure to 10 and 30 Gy of gamma, significantly decreased ASCs viability in comparison to the non-irradiated group. We also showed that 5 Gy of irradiation caused significant upregulation of ABCA1 as well as MMP-9 in ASCs. Accordingly, different doses of radiation may lead to differential changes in ASCs located in TME, which might affect radiotherapy outcomes.

Given that the viability of cells denotes cells ability to endure external stressors, we studied 
the effects of different doses of gamma irradiation on the ASCs viability at different time points. Our results indicated that, regarding non-irradiated ASCs, increasing of gamma irradiation from 10 to $30 \mathrm{~Gy}$, decreases the viability of ASCs significantly. No significant change was observed in the viability of ASCs following exposure to 5 Gy of gamma at different time points. In this regard, Wang et al. reported that exposure to $\gamma$-irradiation at different doses from 0.25 to 10 Gy of irradiation reduced MSCs viability significantly. In comparison to our study, they used bone marrow sources for extracting MSCs and MTT assay to assess cell viability in mesenchymal stem cells. They showed that the adipogenic and osteogenic differentiation ability of MSCs reduced after irradiation [19]. Moreover, Filion et al. suggested that 5 Gy of irradiation decreases cell viability by approximately $65 \%$ in human embryonic stem cells [20]. It is supposed that gamma irradiation damages DNA in irradiated cells and inhibits the repair system, thereby reducing cell viability. However, it seems that the sources of stem cells are important for their reactions to the various doses of gamma irradiation.

It has been reported that an essential mechanism through which the cells overcome against chemotherapy drugs is ATP-binding cassette (ABC) transporters [21]. Furthermore, the association between the ABC transporters and radiation resistance has been proved [22]. In our study, we showed that 5 Gy but not 10 and 30 Gy of gamma irradiation significantly increased the mRNA expression of ABCA1 in ASCs. In this regard, studies reported that irradiation increase the expression of $\mathrm{ABCA} 1$ in different cell lines [22, 23]. Based on these studies, the release of ABCA1 increases the survival of the cells after irradiation. In addition, the previous study reported that $A B C$ transporters protect embryonic cells against the destructive effects of non-ionizing radiation. In this study, Leite et al. showed that ABCB1 transporter inhibition increased the harmful effects of ultraviolet light in embryonic cells [24]. Although the exact mechanism of ABCA1 upregulation post-irradiation remains to be elucidated, oxidative stress was considered as the most likely mechanism stimulating $\mathrm{ABC}$ transporter expression after irradiation [25].

Several studies demonstrated that irradiation increases MMP-9 protein in epithelial and macrophage cell lines [26, 27]. Studies have also shown that irradiation caused growth and development in cancer cells by increasing the expression of MMP-9 [28, 29]. Our results showed up-regulation of MMP-9 in ASCs after 5 Gy of gamma irradiation. In this regard, Cruet-Hennequart reported that 5 Gy of X-irradiation increased MMP-1, MMP-3 and MMP-13 proteins in human mesenchymal stem cells [30]. It seems that, besides the dose of irradiation, the source of irradiation might also affect the expression of different proteins.

\section{Conclusion}

In light of our findings, gamma irradiation increases the expression of ABCA1 and MMP-9 in ASCs. Accordingly, it can be predicted that the expression of such molecules denotes angiogenic and reconstructive behavior of ASCs. Because of the vicinity of ASCs to the tumor cells in the TME and their radiosensitivity, the response of cancer cells to radiation treatment might be modified by the bystander effects of ASCs, thereby affecting the radiation outcome. Thus, deep insight into TME affords a chance to achieve efficient strategies overcoming tumors during radiation treatment.

\section{Acknowledgment}

The authors would like to thank the patients and all the participants for their kind contribution to this project. This research was extracted from Shahram Taeb's thesis. Also, this work was financially supported by Shiraz University of Medical Sciences, Shiraz, Iran (Grant no.12468) and Shiraz Institute for Cancer Research (Grant no. ICR-100-504) for fulfilling his $\mathrm{PhD}$ degree. 


\section{Authors' Contribution}

S. Taeb Performed all in vitro experiments, analyzed the data and wrote the manuscript. MA. Mosleh-Shirazi Performed in vivo experiment and analyzed the in vivo data. A. Ghaderi, M. Razmkhah and SMJ. Mortazavi Contributed to the concept and design, supervising the data analyses, financial support, and final approval of the manuscript. All authors read and approved the final manuscript.

\section{Ethical Approval}

Ethical approval was obtained by the Ethics Committee of our University (Ethics code: IR.SUMS.REC.1396. S.290).

\section{Informed consent}

Prior to study, participation consent and approval were obtained from five healthy participants recruited for mammoplasty surgery.

\section{Conflict of Interest}

None

\section{References}

1. Reina-Campos M, Shelton PM, Diaz-Meco MT, Moscat J. Metabolic reprogramming of the tumor microenvironment by p62 and its partners. Biochim Biophys Acta Rev Cancer. 2018;1870:8895. doi: 10.1016/j.bbcan.2018.04.010. PubMed PMID: 29702207. PubMed PMCID: PMC6193563.

2. Chen ZY, Hu YY, Hu XF, Cheng LX. The conditioned medium of human mesenchymal stromal cells reduces irradiation-induced damage in cardiac fibroblast cells. J Radiat Res. 2018;59:55564. doi: 10.1093/jrr/rry048. PubMed PMID: 30010837. PubMed PMCID: PMC6151644.

3. Aghaalikhani N, Rashtchizadeh N, Shadpour P, Allameh A, Mahmoodi M. Cancer stem cells as a therapeutic target in bladder cancer. J Cell Physiol. 2019;234:3197-206. doi: 10.1002/jcp.26916. PubMed PMID: 30471107.

4. Zuk PA, Zhu M, Mizuno H, Huang J, et al. Multilineage cells from human adipose tissue: implications for cell-based therapies. Tissue Eng. 2001;7:21128. doi: 10.1089/107632701300062859. PubMed PMID: 11304456.

5. Seo BM, Miura M, Gronthos S, Bartold PM, et al. Investigation of multipotent postnatal stem cells from human periodontal ligament. Lan- cet. 2004;364:149-55. doi: 10.1016/S01406736(04)16627-0. PubMed PMID: 15246727.

6. Sabatini F, Petecchia L, Tavian M, Jodon De Villeroche V, Rossi GA, Brouty-Boye D. Human bronchial fibroblasts exhibit a mesenchymal stem cell phenotype and multilineage differentiating potentialities. Lab Invest. 2005;85:962-71. doi: 10.1038/ labinvest.3700300. PubMed PMID: 15924148.

7. Shi Y, Du L, Lin L, Wang Y. Tumour-associated mesenchymal stem/stromal cells: emerging therapeutic targets. Nat Rev Drug Discov. 2017;16:3552. doi: $10.1038 / n r d .2016 .193$. PubMed PMID: 27811929.

8. Fan W, Li J, Wang Y, Pan J, Li S, Zhu L, et al. CD105 promotes chondrogenesis of synovium-derived mesenchymal stem cells through Smad2 signaling. Biochem Biophys Res Commun. 2016;474:33844. doi: 10.1016/j.bbrc.2016.04.101. PubMed PMID: 27107692.

9. Liu J, Kuwabara A, Kamio Y, Hu S, et al. Human Mesenchymal Stem Cell-Derived Microvesicles Prevent the Rupture of Intracranial Aneurysm in Part by Suppression of Mast Cell Activation via a PGE2-Dependent Mechanism. Stem Cells. 2016;34:2943-55. doi: 10.1002/stem.2448. PubMed PMID: 27350036. PubMed PMCID: PMC5287411.

10. Nwabo Kamdje AH, Kamga PT, Simo RT, et al. Mesenchymal stromal cells' role in tumor microenvironment: involvement of signaling pathways. Cancer Biol Med. 2017;14:129-41. doi: 10.20892/j.issn.2095-3941.2016.0033. PubMed PMID: 28607804. PubMed PMCID: PMC5444925.

11. Corsten MF, Shah K. Therapeutic stem-cells for cancer treatment: hopes and hurdles in tactical warfare. Lancet Oncol. 2008;9:376-84. doi: 10.1016/S1470-2045(08)70099-8. PubMed PMID: 18374291.

12. Tian LL, Yue W, Zhu F, Li S, Li W. Human mesenchymal stem cells play a dual role on tumor cell growth in vitro and in vivo. J Cell Physiol. 2011;226:1860-7. doi: 10.1002/jcp.22511. PubMed PMID: 21442622.

13. Binnewies M, Roberts EW, Kersten K, Chan V, et al. Understanding the tumor immune microenvironment (TIME) for effective therapy. Nat Med. 2018;24:541-50. doi: 10.1038/s41591-0180014-x. PubMed PMID: 29686425. PubMed PMCID: PMC5998822.

14. Arnold KM, Flynn NJ, Raben A, Romak L, et al. The Impact of Radiation on the Tumor Microenvironment: Effect of Dose and 
Fractionation Schedules. Cancer Growth Metastasis. 2018;11:1179064418761639. doi: 10.1177/1179064418761639. PubMed PMID: 29551910. PubMed PMCID: PMC5846913.

15. Barker HE, Paget JT, Khan AA, Harrington KJ. The tumour microenvironment after radiotherapy: mechanisms of resistance and recurrence. Nat Rev Cancer. 2015;15:409-25. doi: 10.1038/ nrc3958. PubMed PMID: 26105538. PubMed PMCID: PMC4896389.

16. Jamal $M$, Rath BH, Williams ES, Camphausen $\mathrm{K}$, Tofilon PJ. Microenvironmental regulation of glioblastoma radioresponse. Clin Cancer Res. 2010;16:6049-59. doi: 10.1158/1078-0432.CCR10-2435. PubMed PMID: 21037023. PubMed PMCID: PMC3005074.

17. Gu H, Huang T, Shen Y, Liu Y, Zhou F, Jin Y, et al. Reactive Oxygen Species-Mediated Tumor Microenvironment Transformation: The Mechanism of Radioresistant Gastric Cancer. Oxid Med Cell Longev. 2018;2018:5801209. doi: 10.1155/2018/5801209. PubMed PMID: 29770167. PubMed PMCID: PMC5892229.

18. Razmkhah $M$, Jaberipour $M$, Hosseini $A$, Safaei A, Khalatbari B, Ghaderi A. Expression profile of IL-8 and growth factors in breast cancer cells and adipose-derived stem cells (ASCs) isolated from breast carcinoma. Cell Immunol. 2010;265:805. doi: 10.1016/j.cellimm.2010.07.006. PubMed PMID: 20705284.

19. Wang Y, Zhu G, Wang J, Chen J. Irradiation alters the differentiation potential of bone marrow mesenchymal stem cells. Mol Med Rep. 2016;13:21323. doi: $10.3892 / \mathrm{mmr} .2015 .4539$. PubMed PMID: 26572960. PubMed PMCID: PMC4686093.

20. Filion TM, Qiao M, Ghule PN, Mandeville M, et al. Survival responses of human embryonic stem cells to DNA damage. J Cell Physiol. 2009;220:58692. doi: 10.1002/jcp.21735. PubMed PMID: 19373864. PubMed PMCID: PMC2925401.

21. Chen Z, Shi T, Zhang L, Zhu P, Deng M, Huang $C$, et al. Mammalian drug efflux transporters of the ATP binding cassette $(A B C)$ family in multidrug resistance: A review of the past decade. Cancer Lett. 2016;370:153-64. doi: 10.1016/j.canlet.2015.10.010. PubMed PMID: 26499806.

22. Ingram WJ, Crowther LM, Little EB, Freeman R, et al. $A B C$ transporter activity linked to radiation resistance and molecular subtype in pediatric medulloblastoma. Exp Hematol Oncol. 2013;2:26. doi: 10.1186/2162-3619-2-26. PubMed PMID:
24219920. PubMed PMCID: PMC3851566.

23. Efferth T, Langguth P. Transport processes of radiopharmaceuticals and-modulators. Radiat Oncol. 2011;6:59. doi: 10.1186/1748-717X-659. PubMed PMID: 21645349. PubMed PMCID: PMC3141524.

24. Leite JC, De Vasconcelos RB, Da Silva SG, De Siqueira-Junior JP, Marques-Santos LF. ATPbinding cassette transporters protect sea urchin gametes and embryonic cells against the harmful effects of ultraviolet light. Mol Reprod Dev. 2014;81:66-83. doi: 10.1002/mrd.22283. PubMed PMID: 24254332.

25. Ishikawa $T$, Nakagawa $H$, Hagiya $Y$, Nonoguchi $N$, Miyatake S, Kuroiwa T. Key Role of Human ABC Transporter ABCG2 in Photodynamic Therapy and Photodynamic Diagnosis. Adv Pharmacol Sci. 2010;2010:587306. doi: 10.1155/2010/587306. PubMed PMID: 21188243. PubMed PMCID: PMC3003952.

26. Yue H, Hu K, Liu W, Jiang J, Chen Y, Wang R. Role of matrix metalloproteinases in radiationinduced lung injury in alveolar epithelial cells of Bama minipigs. Exp Ther Med. 2015;10:143744. doi: 10.3892/etm.2015.2658. PubMed PMID: 26622503. PubMed PMCID: PMC4578051.

27. Zhou Y, Xia L, He ZS, Ouyang W, Z H, Xie CH. Modulation of matrix metalloproteinase- 9 and tissue inhibitor of metalloproteinase-1 in RAW264.7 cells by irradiation. Mol Med Rep. 2010;3:80913. doi: 10.3892/mmr.2010.326. PubMed PMID: 21472318.

28. Kunigal S, Lakka SS, Joseph P, Estes N, Rao JS. MMP-9 inhibition downregulates radiationinduced NF- $\kappa$ activity leading to apoptosis in breast tumors. Clin Cancer Res. 2008;14:3617. doi: 10.1158/1078-0432.CCR-07-2060. PubMed PMID: 18519796. PubMed PMCID: PMC2410036.

29. Jadhav U, Mohanam S. Response of neuroblastoma cells to ionizing radiation: modulation of in vitro invasiveness and angiogenesis of human microvascular endothelial cells. Int $J$ Oncol. 2006;29:1525-31. PubMed PMID: 17088992. PubMed PMCID: PMC2441915.

30. Cruet-Hennequart S, Drougard C, Shaw G, Legendre F, Demoor M, Barry F, et al. Radiation-induced alterations of osteogenic and chondrogenic differentiation of human mesenchymal stem cells. PLOS One. 2015;10:e0119334. doi: 10.1371/ journal.pone.0119334. PubMed PMID: 25837977. PubMed PMCID: PMC4383487. 